\title{
The Rab5-Rab11 Endosomal Pathway is Required for BDNF-Induced CREB Transcriptional Regulation in Hippocampal Neurons
}

\author{
Andrés González-Gutiérrez, ${ }^{1}{ }^{\circledR}$ Oscar M. Lazo, ${ }^{2}$ and ${ }^{\circledR}$ Francisca C. Bronfman ${ }^{1,3}$ \\ ${ }^{1}$ Department of Physiology, Faculty of Biological Sciences, Pontificia Universidad Católica de Chile, Santiago, Av. Libertador Bernardo O'Higgins \\ 340, 8970117 Chile, ${ }^{2}$ Department of Neuromuscular Diseases and UK Dementia Research Institute, UCL Queen Square Institute of Neurology, \\ University College London, London WC1N 3BG, United Kingdom, and ${ }^{3}$ NeuroSignaling Lab (NESLab), Center for Aging and Regeneration (CARE- \\ UC), Institute of Biomedical Sciences (ICB), Faculty of Medicine, Universidad Andrés Bello, Echaurren 183, 8370146, Santiago, Chile
}

Brain-derived neurotrophic factor (BDNF) is a key regulator of the morphology and connectivity of central neurons. We have previously shown that BDNF/TrkB signaling regulates the activity and mobility of the GTPases Rab5 and Rab11, which in turn determine the postendocytic sorting of signaling TrkB receptors. Moreover, decreased Rab5 or Rab11 activity inhibits BDNF-induced dendritic branching. Whether Rab5 or Rab11 activity is important for local events only or for regulating nuclear signaling and gene expression is unknown. Here, we investigated, in rat hippocampal neuronal cultures derived from embryos of unknown sex, whether BDNF-induced signaling cascades are altered when early and recycling endosomes are disrupted by the expression of dominant-negative mutants of Rab5 and Rab11. The activity of both Rab5 and Rab11 was required for sustained activity of Erk1/2 and nuclear CREB phosphorylation, and increased transcription of a BDNF-dependent program of gene expression containing CRE binding sites, which includes activity-regulated genes such as Arc, Dusp1, c-fos, Egr1, and Egr2, and growth and survival genes such as Atf3 and Gem. Based on our results, we propose that early and recycling endosomes provide a platform for the integration of neurotrophic signaling from the plasma membrane to the nucleus in neurons, and that this mechanism is likely to regulate neuronal plasticity and survival.

Key words: BDNF; CREB; dendrites; endosomes; Rab11; Rab5

Significance Statement

BDNF is a neurotrophic factor that regulates plastic changes in the brain, including dendritic growth. The cellular and molecular mechanisms underlying this process are not completely understood. Our results uncover the cellular requirements that central neurons possess to integrate the plasma membrane into nuclear signaling in neurons. Our results indicate that the endosomal pathway is required for the signaling cascade initiated by BDNF and its receptors at the plasma membrane to modulate BDNF-dependent gene expression and neuronal dendritic growth mediated by the CREB transcription factor. CREB is a key transcription factor regulating circuit development and learning and memory.

Received Aug. 25, 2019; revised Sep. 2, 2020; accepted Sep. 8, 2020.

Author contributions: A.G.G., O.M.L., and F.C.B. designed research; A.G.G. performed research; A.G.G., O.M.L., and F.C.B. analyzed data; A.G.G., O.M.L., and F.C.B. wrote the paper.

This research was supported by the ANID (Agencia Nacional de Investigacion y Desarrollo) FONDECYT Grant \#1171137 (F.C.B.), the Basal Center of Excellence in Science and Technology CONICYT (Grant \#AFB170005), the Millennium Nucleus (Grant \#P07/011-F), and fellowships from CONICYT (to A.G.G.), Becas-Chile (0.M.L.), and The Royal Society (0.M.L.). We thank Carolina Ramírez for helping with Western blot quantifications, Anibal Caceres for helping with the qRT-PCR arrays, and Professor Giampietro Schiavo (UCL, London, UK) and Dr. Guillermo Moya-Alvarado (John Hopkins University, Baltimore, MD) for helpful comments on the manuscripts.

A. González-Gutierrez's present address: Department of Neuroscience and Biomedical Neuroscience Institute, Faculty of Medicine, Universidad de Chile, Av Independencia 1027, 8380453, Santiago, Chile.

The authors declare no competing financial interests.

Correspondence should be addressed to Francisca C. Bronfman at francisca.bronfman@unab.cl.

https://doi.org/10.1523/JNEUROSCI.2063-19.2020

Copyright $\odot 2020$ the authors

\section{Introduction}

Neurotrophins shape the nervous system by regulating neuronal morphology and connectivity. Brain-derived neurotrophic factor (BDNF) is the most widely expressed member of this family in the brain and is associated with multiple functions, such as axon growth, dendritic branching, synaptic assembly and neurotransmission (Gonzalez et al., 2016; Kowiański et al., 2018). These diverse roles are explained by the multiplicity of signaling pathways triggered by BDNF. Upon binding of BDNF to tyrosinerelated kinase B (TrkB), the receptor autophosphorylates at specific tyrosine residues in the intracellular domain, triggering the activation of the extracellular signal-regulated kinase 1 (Erk1) and Erk2 and the phosphatidylinositol 3-kinase (PI3K) pathways, among others (Huang and Reichardt, 2003; Minichiello, 2009). 
The signaling events triggered by BDNF/TrkB occur at the plasma membrane, regulating local events such as the protrusion of new neurites (Horch and Katz, 2002; Horch, 2004), and propagate to the nucleus to impact gene expression (Cohen et al., 2011). The propagation and spatiotemporal dynamics of neurotrophin signaling largely depend on the trafficking of activated receptors as cargoes of membrane organelles known as "signaling endosomes" (Barford et al., 2017; Villarroel-Campos et al., 2018). Consistently, endocytosis and trafficking of TrkB are required for activation of the PI3K signaling pathway and are necessary for sustained activation of Erk1/2 (Zheng et al., 2008; Fu et al., 2011; Xu et al., 2016).

The intracellular trafficking of endocytic membranes is regulated by the Rab family of monomeric GTPases. Rab5 is a main determinant of early endosome identity; from there, cargoes can be sorted to Rab7 endosomes and lysosomes or recycled to the plasma membrane via Rab11-positive recycling endosomes (Stenmark, 2009; Jovic et al., 2010; Goldenring, 2015). We showed that TrkB localizes to both Rab5 and Rab11 endosomes after activation by BDNF in dendrites and cell bodies, and both GTPases are critical for BDNF-mediated dendritic branching (Lazo et al., 2013; Moya-Alvarado et al., 2018). While Rab11 has been shown to mediate BDNF/TrkB signaling events in dendrites and synapses (Huang et al., 2013; Lazo et al., 2013), to what extent they contribute to the propagation of BDNF-induced signaling cascades to the nucleus is not well understood.

The cAMP response element-binding protein (CREB) is a transcription factor (TF) constitutively expressed by neurons and is well known for mediating activity-dependent transcription, a process required for the development of neuronal connectivity. CREB exerts its effects by regulating the transcription of immediate-early genes such as c-fos, $A r c, B d n f$, and the Egr family of TFs. The signaling mechanisms upstream of CREB include the activation of neurotransmitter receptors and voltage-gated channels that increase intracellular calcium levels (Flavell and Greenberg, 2008; Yap and Greenberg, 2018). Interestingly, CREB is also activated by $\mathrm{BDNF}$ signaling through $\operatorname{TrkB}$ receptors (Finkbeiner et al., 1997). Thus, CREB increases BDNF expression, and, in turn, BDNF activates the phosphorylation of CREB, suggesting that there is a bidirectional positive feedback loop for CREB activation.

Different lines of evidence suggest that CREB activation by membrane receptors requires signaling from intracellular organelles. We have previously demonstrated that the trafficking of TrkB through the recycling pathway sensitizes hippocampal neurons to $\mathrm{BDNF}$, increasing activation of the transcription factor CREB at lower ligand concentrations (Lazo et al., 2013). Additionally, there are results indicating that protein kinase A (PKA) signaling from intracellular organelles, mediated by thyroid stimulating hormone (TSH) receptors, is required to activate CREB in primary thyroid cells (Godbole et al., 2017). Finally, axonal signaling endosomes are required for CREB activation to induce survival in sympathetic neurons (Riccio et al., 1999; Yamashita and Kuruvilla, 2016).

Here, we hypothesized that disturbing the endosomal pathway by reducing the activity of Rab5 and Rab11 alters the temporal dynamics of BDNF-induced signaling pathways that regulate CREB activation and gene expression. Our results showed that the integrity of early and recycling endosomes is crucial for CREB activation and further BDNF-induced transcriptional regulation and provide new evidence demonstrating that the endocytic route regulates plasma membrane to nuclear signaling in neurons.

\section{Materials and Methods}

Primary culture of hippocampal neurons. Hippocampal neurons were prepared as described previously (Lazo et al., 2013) from embryonic day 18 rat embryos obtained from the animal facility of the Pontificia Universidad Catolica de Chile and were killed under deep anesthesia according to the bioethical protocols of our institution. Briefly, hippocampi were dissected from embryos of unknown sex, dissociated into single cells in HBSS (Thermo Fisher Scientific), resuspended in Minimum Essential Medium supplemented with 10\% horse serum, 20\% D-glucose, and $0.5 \mathrm{~mm}$ glutamine (MEM/HS), and seeded on poly-L-lysine (1 mg/ml; Sigma-Aldrich) at low density for morphometric and cell biology experiments $\left(7000 \mathrm{cells} / \mathrm{cm}^{2}\right)$ or at medium density for molecular biology and biochemical analysis $\left(15,000 \mathrm{cells} / \mathrm{cm}^{2}\right)$. After $4 \mathrm{~h}$, the culture medium was replaced with Neurobasal Medium (Thermo Fisher Scientific) supplemented with 2\% B27 (Thermo Fisher Scientific) and $0.5 \mathrm{~mm}$ glutamine. The proliferation of nonneuronal cells was limited by the use of $0.5 \mu \mathrm{M}$ cytosine arabinoside (Sigma-Aldrich) beginning at $3 \mathrm{~d}$ in vivo (3 DIV).

Transfections and infections. To express exogenous proteins, we transfected hippocampal neurons at 7 DIV using Lipofectamine 2000 (Thermo Fisher Scientific) or transduced them with adenoviruses to achieve infection efficiency $>80 \%$. Experiments were performed after 48 $\mathrm{h}$ in complete medium. Plasmids containing pEGFP-C1 are commercially available from Clontech. Rab5 and Rab11 in the pEGFP-C1 vector were gifts from Prof. Victor Faundez (Emory University, Atlanta, GA) and Prof. Rejji Kuruvilla (John Hopkins University, Baltimore, MD), respectively. A nonphosphorylatable mutant of CREB [S133A (originally RSV CREB M1] was a gift from Prof. Marc Montimy (plasmid \#22 395, Addgene). We used replication-defective serotype 5 adenoviral vectors for expressing EGFP-Rab5 and EGFP-Rab11 DN mutants under the CMV promoter (Ascaño et al., 2009; Escudero et al., 2019).

Western blot. Neurons were washed in PBS and lysed in a solution containing $150 \mathrm{~mm} \mathrm{NaCl}, 50 \mathrm{~mm}$ Tris- $\mathrm{HCl}$, pH 8.0, 2 mм EDTA, 0.1\% SDS, and $1 \%$ Triton X-100, supplemented with phosphatase and protease inhibitors and then treated with SDS-PAGE sample buffer. After electrophoretic transfer, nitrocellulose membranes were incubated with the following antibodies: rabbit anti-p490TrkA, (1:1000; catalog \# 9141, Cell Signaling Technology); rabbit anti-TrkB (1:1000; donated by Ursula Wyneken, Universidad de Los Andes, Santiago, Chile); rabbit anti-pAkt (1:1000; catalog \# 9271; Cell Signaling Technology); rabbit anti-Akt (1:1000; catalog \#H-136, Santa Cruz Biotechnology); rabbit anti-pS133 CREB (1:500; catalog \#87G3, Cell Signaling Technology); mouse anti-CREB (1:500; catalog \#86B10, Cell Signaling Technology); rabbit anti-pT202/ pY204 ERK1/2 (1:1000; catalog \#9101; Cell Signaling Technology); rabbit anti-Erk1/2 (1:1000; catalog \#9102, Cell Signaling Technology); rabbit antipY515 TrkB (1:1000; catalog \#SAB4300255, Sigma-Aldrich); and mouse anti- $\beta$ III Tubulin (1:1000; catalog \#T8578, Sigma-Aldrich). After incubation with the respective secondary antibodies, membranes were developed with SuperSignal Pico West Chemiluminescent Substrate (catalog \#34080, Thermo Fisher Scientific).

Immunofluorescence. Neurons were washed in PBS in the presence of phosphatase inhibitors (from here on, the inhibitors were always present in the buffer) and then fixed for $15 \mathrm{~min}$ in $3 \%$ PFA and $4 \%$ sucrose dissolved in PBS. Next, the cells were incubated in $0.15 \mathrm{M}$ glycine dissolved in PBS for $10 \mathrm{~min}$ and then blocked and permeabilized simultaneously by incubation for $1 \mathrm{~h}$ in $4 \%$ BSA and $0.5 \%$ Triton X-100 in PBS. Incubation with primary antibodies occurred overnight at $4^{\circ} \mathrm{C}$. Primary antibodies were diluted in $2 \%$ BSA, $0.1 \%$ Triton X-100, $0.1 \mathrm{mM} \mathrm{CaCl}_{2}$, and $1.5 \mathrm{mM} \mathrm{MgCl}_{2}$ dissolved in PBS at the following concentrations: anti-MAP2, 1:200 (catalog \#MAB3418, EMD Millipore); anti-phosphorylated CREB (pCREB), 1:750 (catalog \#87G3, Cell Signaling Technology); anti-pErk1/2, 1:400 (catalog \#9101, Cell Signaling Technology); and rabbit anti-Rab11, 1:200 (catalog \#71-5300, Zymed/Thermo Fisher Scientific). Then, neurons were washed three times with PBS and incubated for 90 min with Alexa Fluor-conjugated secondary antibodies 1:400 (Thermo Fisher Scientific) in a solution with a composition identical to that used for primary antibodies. Finally, coverslips were stained for $10 \mathrm{~min}$ with $1 \mu \mathrm{g} / \mathrm{ml}$ Hoechst stain (catalog \#33285, Thermo Fisher Scientific), washed in PBS and distilled water, and mounted with Mowiol 4-88 (Sigma-Aldrich) on microscope slides. 
Immunoendocytosis for TrkB receptors and transferrin internalization. Immunoendocytosis for recombinant TrkB-Flag receptors wasperformed based on our previous work (Lazo et al., 2013). Briefly, hippocampal neurons (7 DIV) were cotransfected by using Lipofectamine 2000 with plasmids driving the expression of EGFP or Rab5DN- and TrkB-Flag-tagged receptor. At 9 DIV, neurons were incubated for $3 \mathrm{~h}$ in Neurobasal Medium in the presence of $200 \mathrm{ng} / \mathrm{ml}$ TrkB-fc to deplete endogenous BDNF. Then, neurons were incubated at $4^{\circ} \mathrm{C}$ for $30 \mathrm{~min}$ in the presence of anti-Flag antibody conjugated to Alexa Fluor 594 in Neurobasal Medium. Then, neurons were rinsed with warm Neurobasal Media and stimulated or not with BDNF $(50 \mathrm{ng} / \mathrm{ml})$ for $15 \mathrm{~min}$. In another experimental group, neurons were incubated for $3 \mathrm{~h}$ in Neurobasal Medium and then incubated with Alexa Fluor 647-labeled transferrin (catalog \#T23366, Thermo Fisher Scientific) at a $100 \mu \mathrm{g} / \mathrm{ml}$ final concentration for $90 \mathrm{~min}$. Finally, neurons were washed with warm PBS and fixed as described above.

Analysis of BDNF-induced increases in pCREB and pErk1/2. Hippocampal neurons were starved for $3 \mathrm{~h}$ and stimulated with BDNF for different times points at $37^{\circ} \mathrm{C}$. Serum and BDNF deprivation was induced by incubating the neurons with $200 \mathrm{ng} / \mathrm{ml}$ TrkB-Fc (catalog \#688TK, R\&D Systems) in Neurobasal Medium. When indicated, neurons were treated for $30 \mathrm{~min}$ before BDNF stimulation with $30 \mu \mathrm{M}$ PD98059 (Promega), $10 \mu \mathrm{M}$ LY294002 (Calbiochem), or 200 nм K252a (Santa Cruz Biotechnology). At the end of the treatment, neurons were washed in PBS in the presence of phosphatase inhibitors (catalog \#88667, Thermo Fisher Scientific), and levels of total and phosphorylated CREB, as well as total and phosphorylated Erk1/2, were evaluated by using phospho-specific antibodies. In experiments involving microscopy, cells were colabeled with Hoechst 33258 stain for $10 \mathrm{~min}$. Confocal images were analyzed using ImageJ software, and the integrated intensity (sum of the intensity in a region of interest) was measured within the nuclei. Values are expressed as BDNF-induced increase in intensity levels with respect to untreated neurons (time 0 ). When phosphorylation was evaluated by using Western blotting, $50 \mu \mathrm{g}$ of total protein was loaded, and phosphorylation levels were plotted as the intensity of the phosphorylated form with respect to total CREB, TrkB, Akt, or Erk1/2 levels.

Stimulation and measurement of dendritic arborization induced by $B D N F$. Morphologic changes in dendritic arborization induced by BDNF stimulation ( $50 \mathrm{ng} / \mathrm{ml}$; Alomone Labs) were measured in cultured hippocampal neurons as we previously described (Lazo et al., 2013). Briefly, neurons were grown for $7 \mathrm{~d}$ in vitro and then transfected using Lipofectamine 2000 with plasmids driving the expression of EGFP or a nonphosphorylatable mutant of CREB. BDNF was added to the neurons immediately after the transfection protocol was over. After $48 \mathrm{~h}$ of BDNF treatment, neurons were processed for morphologic analysis.

RNA isolation and quantitative real-time PCR. Total RNA was extracted from hippocampal cell cultures using an RNAeasy Mini Kit from Qiagen and then treated with DNase I. To analyze BDNF-induced changes in the levels of $A r c$, we synthesized cDNA starting from $1 \mu \mathrm{g}$ of total RNA, and quantitative real-time PCR (qRT-PCR) was performed with Applied Biosystems SYBR Green (Thermo Fisher Scientific). The custom-made primers used were as follows: for Arc, 5'-GGAGGG AGGTCTTCTACCGT-3' (forward) and $5^{\prime}$-CTACAGAGACAGTGT GGCGG-3' (reverse); for $\beta$-actin, $5^{\prime}$-CCCGCGAGTACAACCTTCT-3' (forward) and $5^{\prime}$-CGTCATCCATGGCGAACT-3' (reverse); for Tbp, 5' -CTGTTTCATGGTGCGTGACGAT-3' (forward) and 5' -AAGCCCT GAGCATAAGGTGGAA-3' (reverse); and for Pgk-1, 5' -TGCTGGGCA AGGATGTTCTGTT-3' (forward) and 5' -ACATGAAAGCGGAGGTT CTCCA-3' (reverse).

Statistical analysis. The results are expressed as the average \pm SEM. Before any statistical testing, datasets were analyzed for normality and homoscedasticity. Sholl analysis curves were compared using repeatedmeasures ANOVA, followed by Bonferroni's multiple-comparison post-test to compare all pairs of datasets. Other differences between treatments were compared by using Student's $t$ test, one-way and twoway ANOVA or Kruskal-Wallis test, depending on the number of groups; and Bonferroni correction was applied to multiple comparisons where appropriate. The specific test used for each dataset, together with its significance and number of replicates, are indicated in the respective figure legend, and all the details, including exact $p$ values and degrees of freedom, have been included in Extended Data 1-1, 2-1, 3-1, 4-1, 5-1, 6-1 supporting Figures 1-6. Statistical analyses were performed using GraphPad Prism 5 (GraphPad Software).

\section{Results}

Sustained BDNF signaling requires Rab5 and Rab11 activity Sustained signaling induced by neurotrophins is required for Erk1/2 nuclear translocation and differentiation of PC12 cells into a neuronal phenotype. However, transient activation of Erk1/2 retains it in the cytoplasm and promotes proliferation (Marshall, 1995). Consistently, sustained activation of Erk1/2 is crucial for structural plasticity in hippocampal neurons (Wu et al., 2001b). We used dominant-negative (DN) mutants of Rab5 and Rab11 to investigate whether early and recycling endosomes play a role in sustained BDNF signaling. Hippocampal neurons (7 DIV) were transduced with control EGFP, Rab5DN, or Rab11DN adenovirus and were stimulated with BDNF $48 \mathrm{~h}$ later for $5 \mathrm{~min}$ to evaluate activation of the signaling pathways or for $120 \mathrm{~min}$ to monitor sustained activation.

Five minutes of stimulation with BDNF led to robust phosphorylation of TrkB, Akt, and Erk1/2, which was unaffected by the presence of mutants Rab5DN and Rab11DN (Fig. 1A,B). Additionally, the levels of total TrkB, Akt, and Erk1/2 remained unaltered after $2 \mathrm{~d}$ of expression of the mutants (Fig. 1A,B). However, after $120 \mathrm{~min}$, the levels of phosphorylated Erk1/2 were still significantly increased in EGFP-expressing neurons, while cells expressing Rab5DN and Rab11DN decreased to basal levels. Akt activity appeared to normally return to basal levels by $120 \mathrm{~min}$ of BDNF stimulation in control cells. Interestingly, neurons expressing the DN mutants showed even lower levels of Akt phosphorylation, suggesting that responsiveness to Akt can be progressively compromised when early and recycling endosomes are disrupted.

The Rab5 GTPase is the first Rab protein regulating postendocytic events of ligand-mediated internalization of receptors (Langemeyer et al., 2018). To investigate whether the downregulation of Rab5 activity affects the BDNF-mediated internalization of TrkB, we performed an immunoendocytosis assay as described previously (Lazo et al., 2013). We found that the expression of the Rab5 DN mutant did not reduce the internalization of $\operatorname{TrkB}$ receptors compared with neurons expressing EGFP (Fig. 2A). We also studied whether the internalization of transferrin, a general marker of clathrin-mediated internalization, was altered by the expression of the Rab5DN mutant. We found that transferrin internalization in cells expressing Rab5DN was comparable to the levels of transferrin internalization in EGFP-expressing neurons (Fig. 2B). These results indicate that expressing the Rab5DN mutant did not impair ligand-mediated internalization in hippocampal neurons compared with EGFPexpressing cells. Therefore, the effects of reducing the activity of Rab5 and Rab11 on TrkB signaling are likely a consequence of altered postendocytic trafficking and not because of reduced expression of TrkB, Akt, or Erk or reduced TrkB internalization. Supporting this idea, we found that the expression of Rab5DN negatively regulates the expression of endogenous Rab11 in the soma and dendrites (Fig. 2C), indicating that the expression of Rab11 depended directly or indirectly on the activity of Rab5.

\section{Rab5 and Rab11 activity is required for BDNF-induced CREB and Erk1/2 phosphorylation in the nucleus}

Several lines of evidence indicate that BDNF signaling activates the transcription factor CREB (Kwon et al., 2011). Additionally, 
A

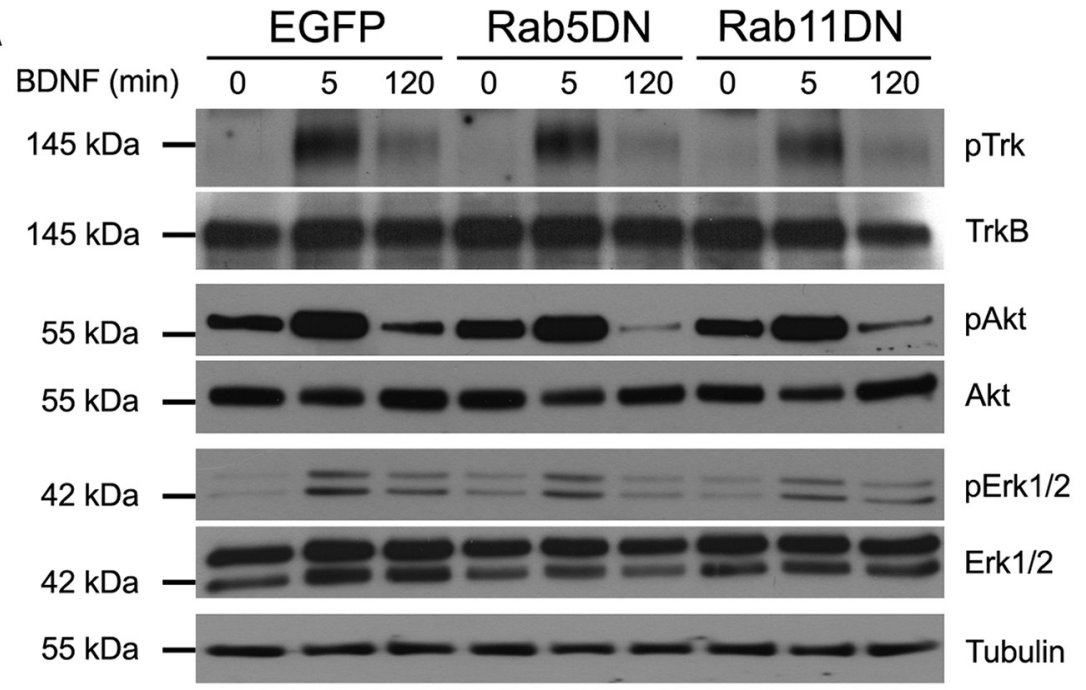

B

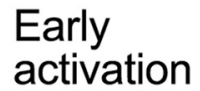

Sustained activation
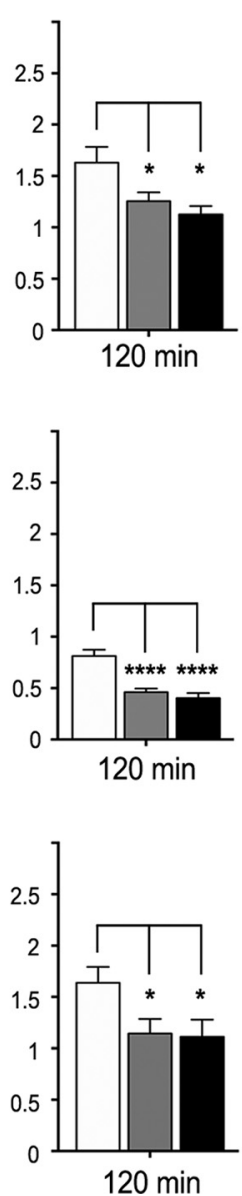

Total
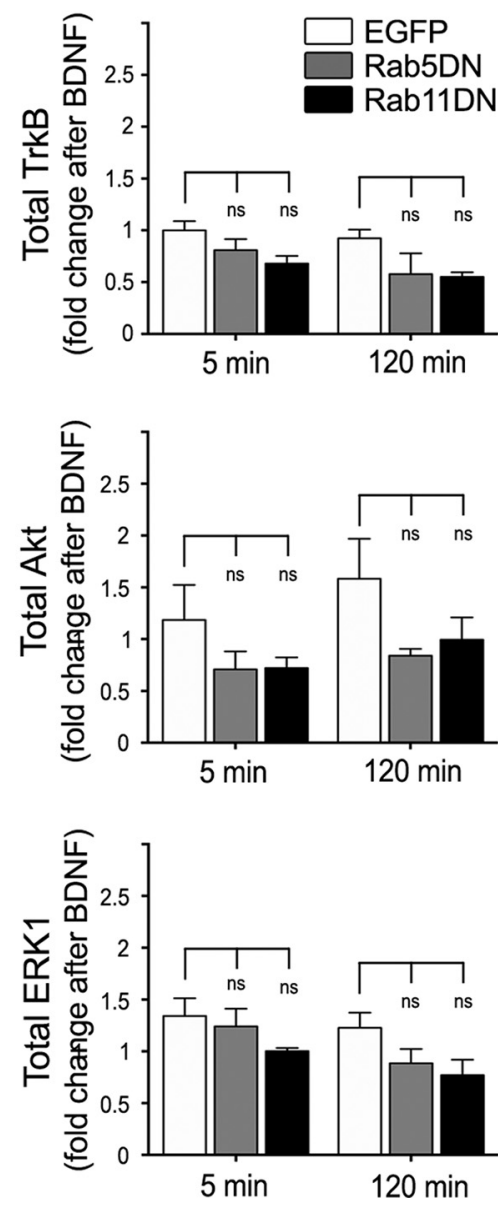

Figure 1. Rab5 and Rab11 activity is required for sustained BDNF downstream signaling. Hippocampal neurons expressing control EGFP or DN mutants of Rab5 and Rab11 were stimulated with BDNF for 0, 5, or 120 min, and lysates were probed for total and phosphorylated TrkB (Y515), Akt (S473), and Erk1/2 (T202/Y204). A, B , Representative Western blots (A) and quantification of five to seven independent experiments $(\boldsymbol{B})$ are shown. The first two columns, "Early activation" and "Sustained activation," were statistically analyzed by using one-way ANOVA, followed by Bonferroni's multiple-comparison post-test, indicated in brackets. The third column was analyzed by two-way ANOVA, which showed no statistically significant differences. Bonferroni's multiple-comparison post-test was applied to compare Rab5DN and Rab11DN against EGFP controls. No significant differences were found. ${ }^{*} p<0.05 ;{ }^{* * *} p<0.0001$; ns, nonsignificant. A summary of the statistics for this figure, including tests, degrees of freedom, and exact $p$ values, has been included in Extended Data Figure 1-1 supporting Figure 1. the results presented above (Fig. 1) indicate that TrkB-sustained signaling is impaired when Rab5 and Rab11 activity is downregulated. Thus, we investigated whether Rab5 and Rab11 regulate CREB activation in the nucleus. For this aim, we transduced 7 DIV hippocampal neurons with control EGFP, Rab5DN, or Rab11DN adenoviral vectors. After $48 \mathrm{~h}$, cells were stimulated with BDNF for $15 \mathrm{~min}$ and compared with nonstimulated controls, and the levels of phosphorylated CREB at the S133 residue were evaluated by Western blotting (Fig. 3A). EGFP-expressing neurons responded to BDNF stimulation with a significant increase in PCREB levels, while the expression of DN mutants of Rab5 and Rab11 completely blocked this effect (Fig. $3 A, B)$. To confirm this observation and further investigate the spatiotemporal dynamics of CREB activation, we used confocal microscopy to evaluate phosphorylated CREB in the nuclei of neurons by immunofluorescence. Neurons expressing control EGFP or the DN mutants of Rab5 and Rab11 were stimulated for $0,15,30$, or 60 min with BDNF. While control EGFP-expressing neurons showed a strong increase in CREB activation after 15-60 min of stimulation (Fig. $3 C-F$ ), the expression of Rab5DN and Rab11DN significantly blocked BDNFinduced activation of CREB in the nucleus (Fig. 3C-F). After 60 min of BDNF stimulation, there was a tendency for increased activation of CREB phosphorylation in neurons expressing the $\mathrm{DN}$ mutants compared with EGFP. However, it did not reach statistically significant levels (Fig. 3E,F).

In different models, CREB can be activated through the Ras-Erk and PI3KAkt signaling pathways (Finkbeiner et al., 1997; Du and Montminy, 1998). Since both signaling pathways are downstream of BDNF/TrkB, we decided to evaluate their contribution to the activation of CREB in our system. To achieve this aim, we used 9 DIV hippocampal neurons treated with a selective inhibitor of Erk1/ 2 PD98059 (Murray et al., 1998) or a potent general inhibitor of PI3K LY294002 (Leemhuis et al., 2004), and we stimulated these cells with BDNF for $15 \mathrm{~min}$. The activation of CREB in the nucleus was measured by using immunofluorescence (Fig. 3G,H). BDNF stimulation induced a strong increase in nuclear pCREB, which was blocked by the Trk inhibitor K252a (Tapley et al., 1992). Neurons treated with PD98059 showed 
robust inhibition of CREB activation in the nucleus (Fig. 3G,H). In contrast, the presence of LY294002 did not alter the BDNFinduced activation of CREB, suggesting that the PI3K-Akt cascade is dispensable to induce CREB activation in hippocampal neurons at this time point. To monitor whether LY294002 interfered with the activation of Akt without any effect on TrkB, we examined the phosphorylation of both proteins by Western blot (Fig. $3 I$ ). We confirmed that although BDNFdependent TrkB phosphorylation was independent of the presence of the inhibitor, Akt phosphorylation at serine 473 was completely blocked. Therefore, BDNF-induced nuclear phosphorylation of CREB essentially depends on Erk1/2 activation in our model.

To investigate to what extent sustained activation of Erk1/2 was affected at specific neuronal compartments (dendrites, cell bodies, and nuclei), we performed immunofluorescence to detect phosphorylated T202 or Y204 of Erk1/2 (pErk1/2). We treated hippocampal neurons with BDNF for $60 \mathrm{~min}$. At this time point, we observed increased phosphorylation of CREB in response to BDNF in neurons expressing EGFP and a tendency to increase levels in the Rab5 and Rab11 mutants (Fig. 3E,F). Treatment of control neurons with BDNF increased the $\mathrm{pErk1/2}$-associated fluorescence in the nucleus, cell body, and dendrites of hippocampal neurons, and, consistent with the results shown in Figure 1, neurons expressing Rab5DN or Rab11DN and stimulated with BDNF for $60 \mathrm{~min}$ showed a decreased response to BDNF (Fig. 4A,B). Fluorescence intensity was quantified in dendrites, somas, and nuclei, and expression of Rab5DN significantly reduced pErk1/2-associated fluorescence in all these neuronal compartments. Although the expression of Rab11DN did not reduce pErk $1 / 2$ in dendrites, the activity in the soma was significantly decreased and largely eliminated in the nucleus by this mutant (Fig. $4 A, B$ ). The staining was abolished in the soma and nucleus when cells were cotreated with BDNF and the Erk1/2 inhibitor PD98059 (Fig. 4C,D), demonstrating the specificity of the staining. Altogether, these data indicate that recycling endosomes are required to propagate Erk1/ 2-CREB phosphorylation to the nucleus.

In summary, our results suggest that, although activation of TrkB, Akt, and Erk1/2 can occur normally in neurons
A
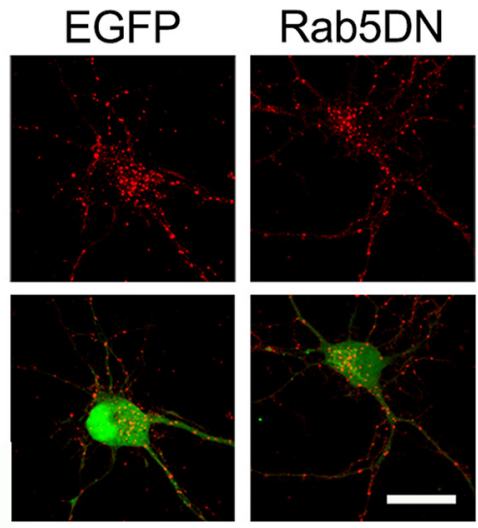

internalized TrkB-Flag $\odot$ EGFP

B
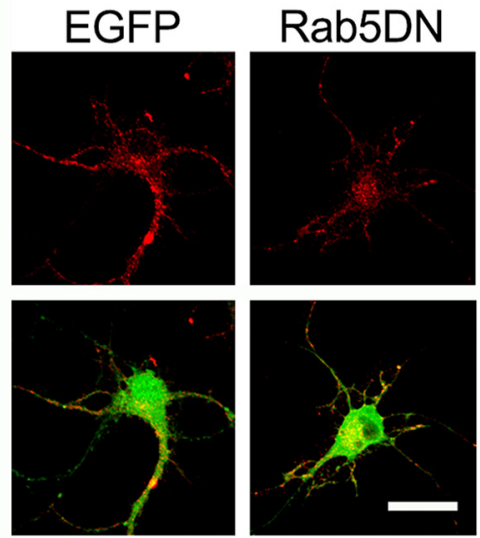

internalized Transferrin EGFP
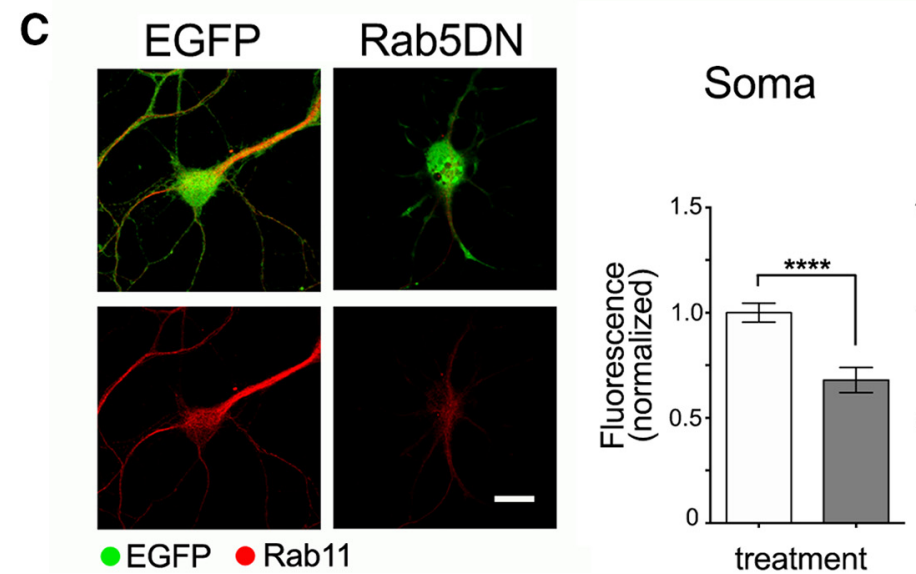

Dendrites
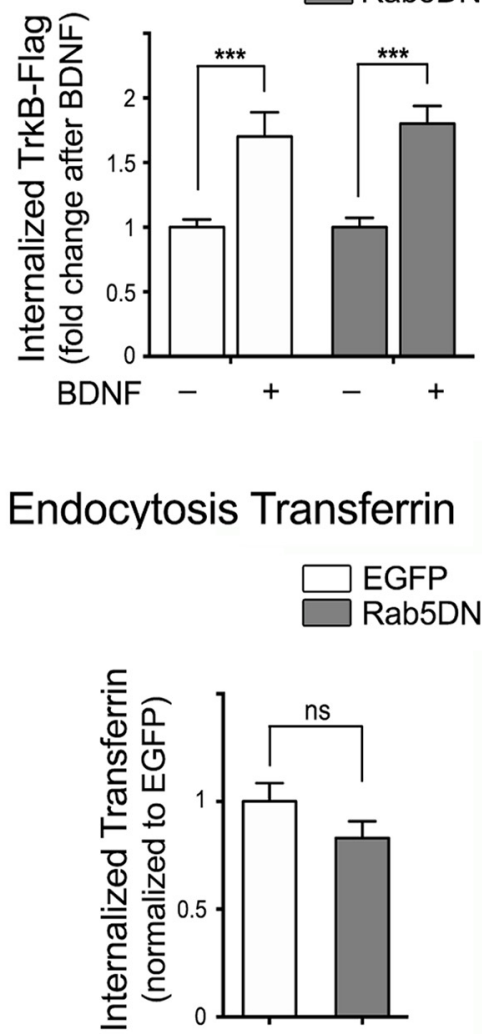

Figure 2. Reduction of Rab5 activity does not decrease internalization of TrkB or transferrin receptor. $\boldsymbol{A}$, The level of endocytosis of TrkB-Flag on stimulation with BDNF in neurons expressing Rab5DN mutant or EGFP as a control. Intermalized TrkB is expressed as the fold change after BDNF and the fluorescence associated with the soma of 17-25 neurons/condition from three independent experiments. BDNF treatment accounted for the majority of the variation, and no effect of the mutant was found $(p=0.6788)$. Data were analyzed by using two-way ANOVA and Bonferroni's multiple-comparison post-test revealed a significant effect of BDNF on endocytosis in both EGFPand Rab5DN-expressing neurons $(* * * 0,001)$. B, Quantification of fluorescently labeled transferrin internalization is also shown as a control. The fluorescence associated with the soma of 16 neurons per condition from three independent experiments was considered for quantification. No significant differences were found between EGFP- and Rab5DN-expressing neurons $(p=0.1499)$. Scale bar, $20 \mu \mathrm{m}$. C, The effects of expressing EGFP and Rab5DN on the endogenous levels of Rab11 were studied by immunofluorescence using a polyclonal antibody against Rab11. Hippocampal neurons were transduced at 7 DIV and analyzed after $48 \mathrm{~h}$. Left, EGFP/Rab5DN expression is shown in green, and Rab11 expression is shown in red. Right, Levels of Rab11 were measured in the soma and dendrites of hippocampal neurons (9 DIV). For quantification, 22-26 neurons/condition from three independent experiments were considered. Data were analyzed by using Student's $t$ test, and the level of significance is indicated as ${ }^{* * *} p<0.0001$. Scale bars, $20 \mu \mathrm{m}$. A summary of the statistics for this figure, including tests, degrees of freedom, and exact $p$ values, has been included in Extended Data Figure 2-1 supporting Figure 2. 
A

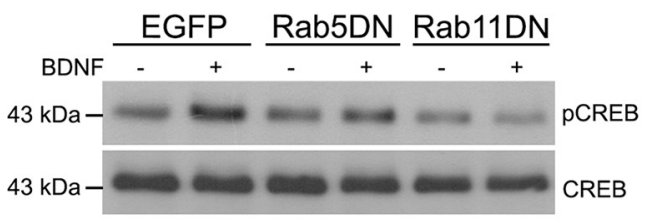

C

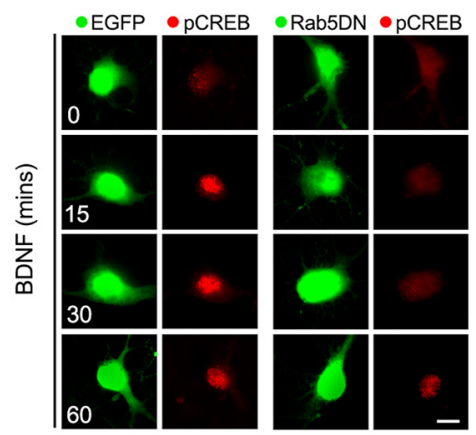

$\mathbf{E}$

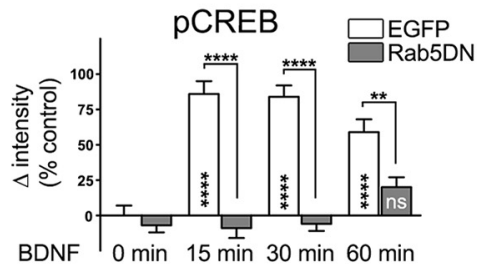

G

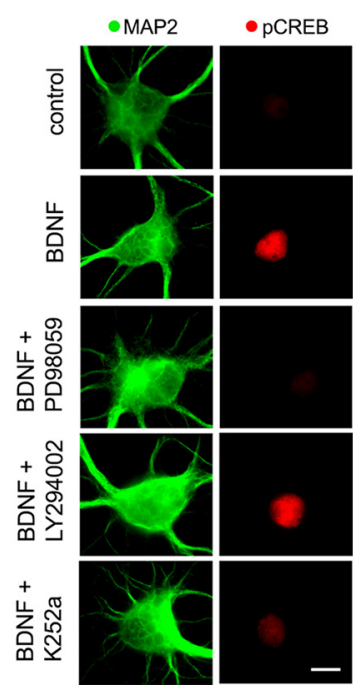

B

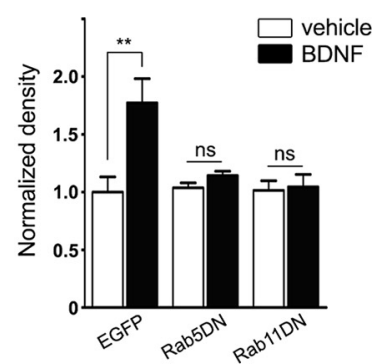

D

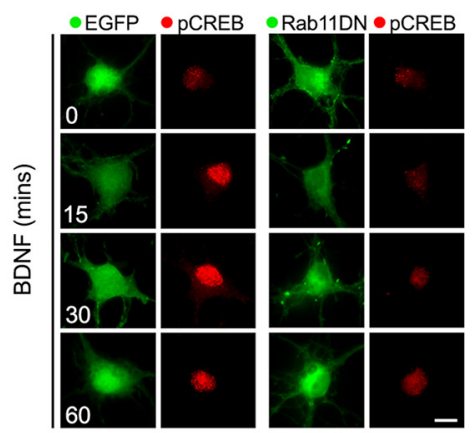

$\mathbf{F}$

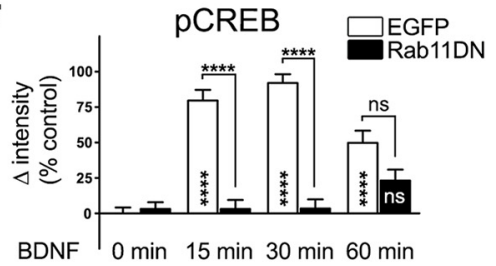

H

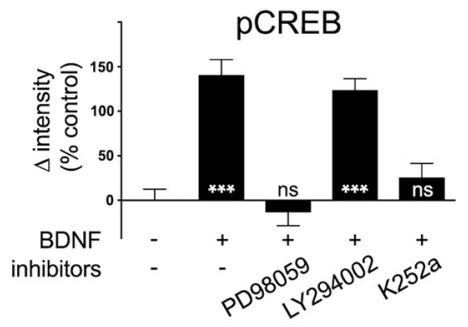

I

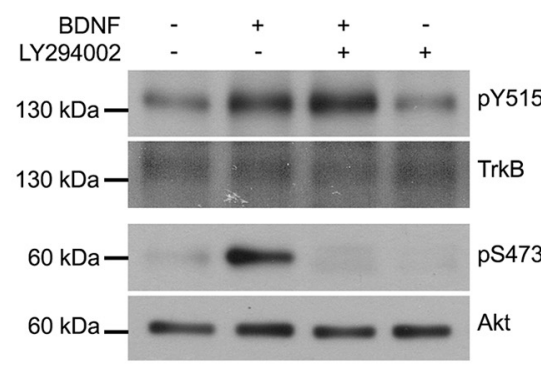

Figure 3. Nuclear CREB phosphorylation requires Rab5 and Rab11 activity. Hippocampal neurons expressing control EGFP or DN mutants of Rab5 and Rab11 were stimulated with BDNF for $15 \mathrm{~min}$, and lysates were probed for total and phosphorylated CREB. $\boldsymbol{A}, \boldsymbol{B}$, Representative Western blots $(\boldsymbol{A})$ and quantification of four independent experiments $(\boldsymbol{B})$ are shown. Data were analyzed by using two-way ANOVA. Bonferroni's multiple-comparison post-test revealed a significant effect of BDNF on endocytosis in EGFP ( $\left.{ }^{* *} p<0.01\right)$. There were no significant differences (ns) found in Rab5DN- or Rab11DN-expressing neurons treated with BDNF compared with untreated neurons. $\mathbf{C}-\boldsymbol{F}$, To specifically study the amount of phosphorylated CREB in the nucleus, we performed stimulation with BDNF for $0,15,30$, or $60 \mathrm{~min}$, and we detected phosphorylated CREB by immunofluorescence. Quantification of normalized intensity from 42 to 112 nuclei/condition from four independent experiments is shown $(\boldsymbol{E}, \boldsymbol{F})$. Datasets were tested by using two-way ANOVA, followed by Bonferroni's multiple-comparison test. Statistically significant differences in $\mathrm{pCREB}$ at different time points compared with time $=0 \mathrm{~min}$ are indicated within the bar. Differences between EGFP controls and mutant-expressing neurons at specific time points are indicated in brackets. Significance levels are labeled ${ }^{* *} p<0.01 ;{ }^{* * *} p<0.001$; and ns, nonsignificant. Scale bars, $10 \mu \mathrm{m}$. G, Hippocampal neurons were stimulated with BDNF for 15 min in the presence of the MEK1/2 inhibitor PD98059, the PI3K inhibitor LY294002, or the Trk kinase activity inhibitor K252a, and were then probed for phosphorylated CREB and MAP2 by immunofluorescence.

expressing DN mutants, sustained signaling by Erk1/2 requires the activity of the small GTPases Rab5 and Rab11, potentially resulting in consequences at the transcriptional level.

CREB phosphorylation is required for BDNF-induced dendritic branching We previously established that Rab5 and Rab11 play a critical role in BDNFinduced dendritic branching (Lazo et al., 2013; Moya-Alvarado et al., 2018), and we showed that both GTPases are required for BDNF-induced CREB phosphorylation (Fig. 3). In addition, BDNF/TrkB signaling regulates Rab5 and Rab11 activity and dynamics, suggesting that both GTPases are downstream targets of BDNF/TrkB signaling (Lazo et al., 2013; Moya-Alvarado et al., 2018). To further corroborate that CREB is required for BDNF-induced dendritic branching, we expressed a nonphosphorylatable CREB mutant (CREB S133A) in 7 DIV hippocampal neurons and stimulated them with BDNF for $48 \mathrm{~h}$ immediately after transfection (Fig. $5 A-C$ ). Since the expression of CREB S133A for $48 \mathrm{~h}$ did not decrease the complexity of dendritic arbors, we concluded that it does not affect basal dendritic morphology at this time point. However, the BDNF-induced increase in complexity, as measured by Sholl analysis (Fig. 5B), and, more specifically, the increase in the number of branches (Fig. 5C) were completely abolished by the presence of the CREB S133A mutant, suggesting that phosphorylation of CREB is needed for BDNF-dependent dendritic branching downstream of TrkB and Erk1/2. Together, our results suggest that $\mathrm{BDNF} / \mathrm{TrkB}$ signaling activates nuclear CREB through the Erk1/2 pathway, requiring an increase in the activities of Rab5 and Rab11 to regulate dendritic branching.

$\boldsymbol{H}$, Quantification of 30 nuclei/condition from three independent experiments showed a complete block of BDNF-induced CREB phosphorylation in the presence of PD89059. Significant differences compared with nonstimulated neurons were analyzed by using one-way ANOVA, followed by Bonferroni's multiple-comparison post-test and are indicated within the bars $* * * * 00.0001$. The dependence on TrkB was confirmed by the robust inhibition of BDNF-induced CREB phosphorylation by K252a. In contrast, LY294002 did not have any effect on pCREB, despite its significant inhibition of Akt phosphorylation (pS473), as shown in $\boldsymbol{G}$ and $\boldsymbol{H}$. $\boldsymbol{I}$, The null effect of LY294002 on TrkB phosphorylation (pY515) is also shown. A summary of the statistics for this figure, including tests, degrees of freedom, and exact $p$ values, has been included in Extended Data Figure 3-1 supporting Figure 3. 
Rab11 activity is required for a distinctive CREB-mediated gene expression pattern in response to BDNF

We have previously shown that Rab5 activity is required for both the maintenance of basal dendritic complexity and for the increase in dendritic complexity induced by BDNF in hippocampal neurons. Rab11DN-expressing neurons, on the other hand, did not show a decrease in basal dendritic complexity at 7 DIV, therefore being specifically required for BDNF-induced dendritic branching (Lazo et al., 2013; Moya-Alvarado et al., 2018). These results indicated that Rab5 has more pleiotropic effects on dendritic morphology than Rab11. During the endocytic sorting of signaling receptors, Rab11 recycling processes are downstream of Rab5-dependent early endosomal sorting. Normally, receptors are internalized to Rab5 early endosomes and then sorted to either Rab11 recycling endosomes or Rab7 late endosomes (Jovic et al., 2010; Bronfman et al., 2014). Additionally, we observed that decreasing Rab5 activity reduced the levels of endogenous Rab11 in dendrites and the somas of hippocampal neurons (Fig. 2C). For these reasons, we decided to focus on the effect of specifically altering the Rab11 recycling endosomal route on CRE-dependent gene expression induced by BDNF since CREB exerts its function by binding specifically to genes targeted by CRE regulatory elements (ChaMolstad et al., 2004).

As shown in Figure 3, reducing Rab5 or Rab11 activity significantly decreases BDNF-induced CREB phosphorylation. However, according to previous reports, despite being a key step, phosphorylation of CREB at serine 133 does not suffice to trigger gene transcription (Zhang et al., 2005; Finsterwald et al., 2010). Therefore, to further address whether the recycling endosomes regulated by concerted activities of Rab5 and Rab11 are required for CREdependent gene expression induced by BDNF, we used a qRT-PCR array for 84 genes regulated by CAMP (CRE), serum (SRE), and $\mathrm{Ca}^{2+}$ (CaRE) response elements. We used 7 DIV hippocampal neurons expressing EGFP or Rab11DN for $2 \mathrm{~d}$ and then stimulated the neurons with BDNF for $4 \mathrm{~h}$. We reliably analyzed 76 genes (Extended Data Fig. 6-2), and we observed that the expression of Rab11DN alone did not reduce or increase the basal levels of expression of the 76 genes included in the array (Extended Data Fig. 6-3). Eleven of these genes were significantly regulated by BDNF after $4 \mathrm{~h}$ of stimulation in EGFP-expressing cells (Fig. $6 \mathrm{~A}$ ).

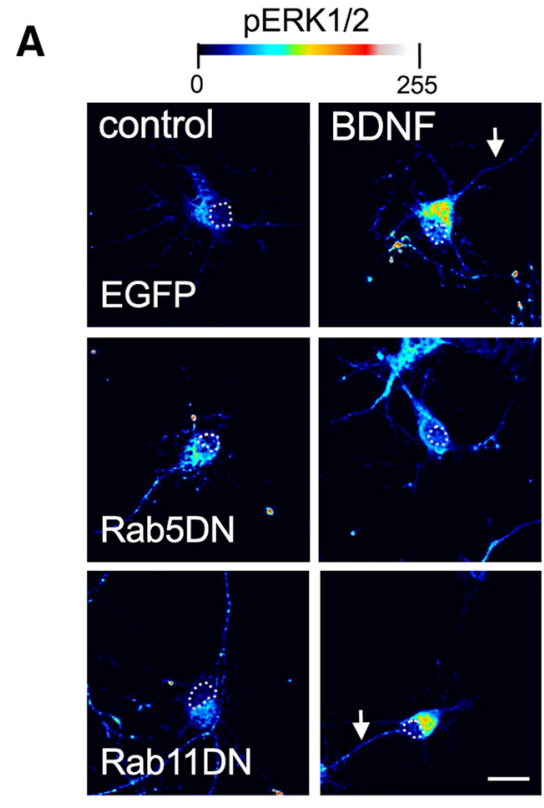

B
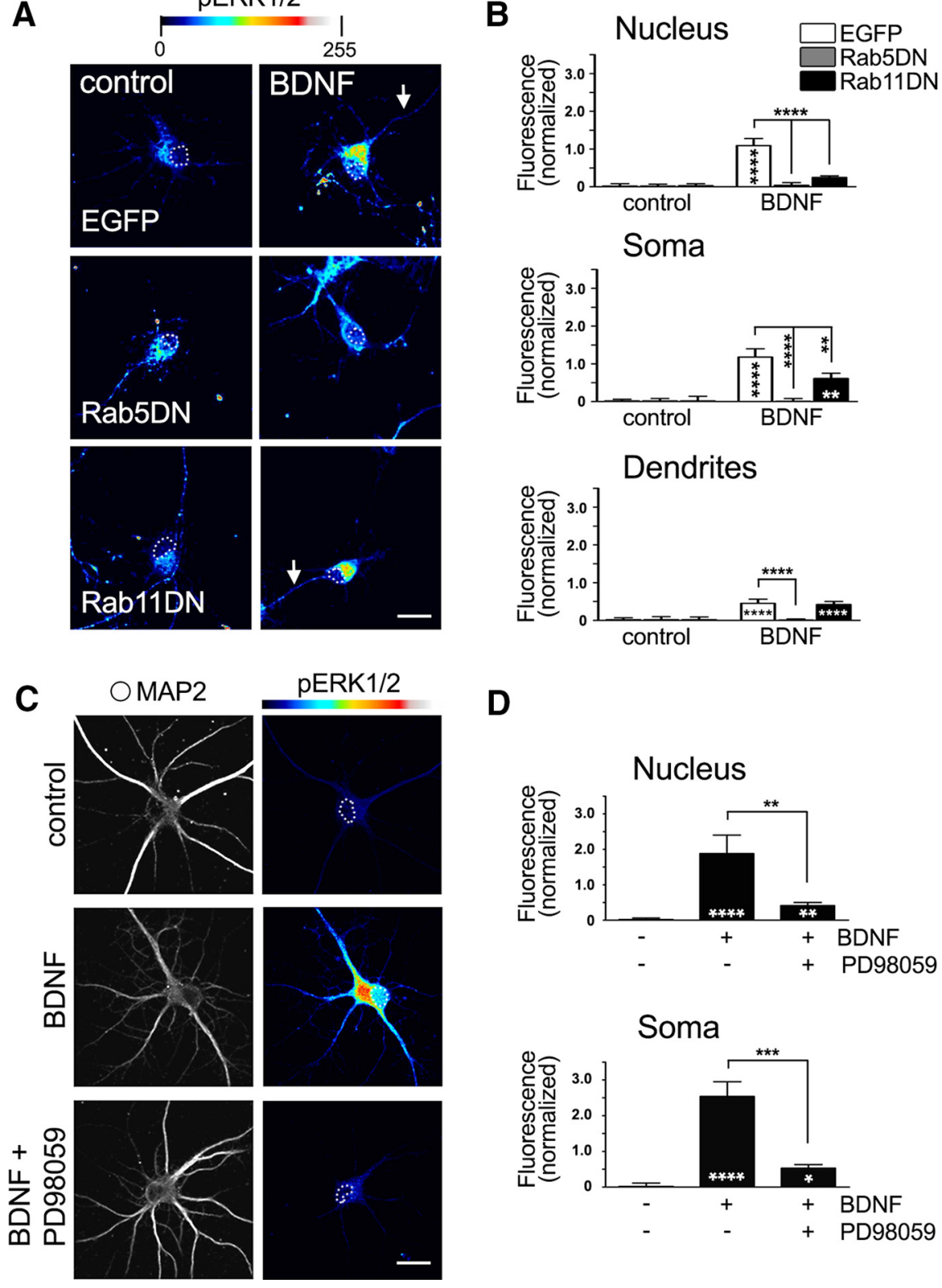

Figure 4. Rab5 and Rab11 activity is required for sustained BDNF-Erk1/2 signaling. $\boldsymbol{A}$, Hippocampal neurons expressing control EGFP or DN mutants of Rab5 and Rab11 were stimulated with BDNF for 0-60 min, and sustained activation of Erk1/2 was analyzed in different neuronal compartments (nuclei, somas, dendrites) by immunofluorescence. Nuclei were traced using Hoechst staining as a reference and are indicated here with a dashed line. Examples of positive dendrites in EGFP and Rab11DN neurons are indicated by white arrowheads. Scale bar, $10 \mu \mathrm{m}$. B, Quantification of normalized intensity from 30 nuclei and somas and 60 dendrites from three independent experiments is shown. In $\boldsymbol{B}$, differences between time $=0$ and 60 min of BDNF stimulation are indicated at the bottom of the bar. The effect of the expression of the Rab5 and Rab11 mutants compared with EGFP after 60 min of BDNF stimulation is indicated in brackets. Statistical differences were tested by using two-way ANOVA, followed by Bonferroni's multiple-comparison test. ${ }^{* *} p<0.01$; ${ }^{* * *} p<0.0001$. C, The ability of PD98059 to inhibit BDNF-induced phosphorylation of Erk1/2 was confirmed by stimulating hippocampal neurons with BDNF for $15 \mathrm{~min}$ and performing double immunofluorescence for MAP2 and pErk1/2. D, Quantification of this effect in somas, dendrites, and nuclei is shown. Quantification of the normalized intensity of the soma and nucleus from 30-45 neurons/condition from three independent experiments is shown. Statistical differences were analyzed by using the Kruskal-Wallis nonparametric test, and Bonferroni's multiple-comparison post-test was applied to analyze differences between groups. ${ }^{*} p<0.05 ;{ }^{* *} p<0.01 ;{ }^{* *} p<0.001 ;{ }^{* * *} p<0.0001$. A summary of the statistics for this figure, including tests, degrees of freedom, and exact $p$ values, has been included in Extended Data Figure 4-1 supporting Figure 4.

The genes significantly modified by BDNF signaling are related to the following neuronal cell responses: neurotransmission (Tacr1 and Th); signaling (Dusp1); neuronal activity and neuronal morphology (Gem); metabolism (Amd1); and transcription control 
A

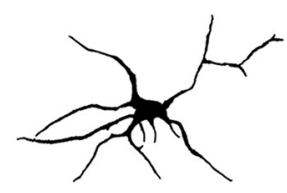

CREB S133A + vehicle
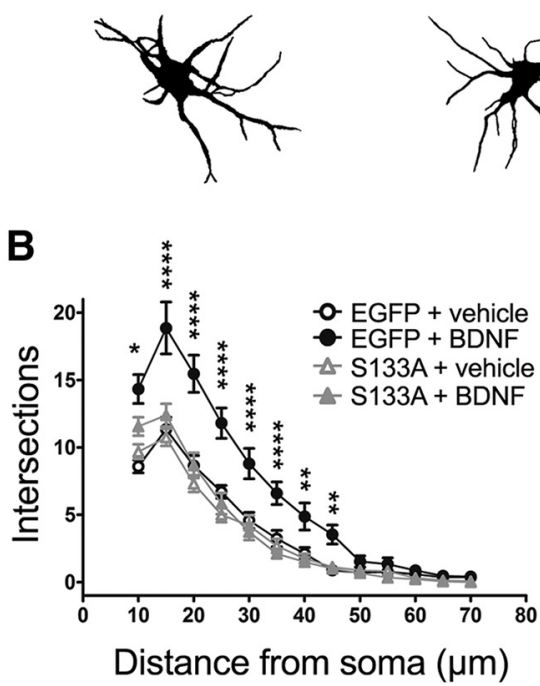

$\mathrm{EGFP}+\mathrm{BDNF}$

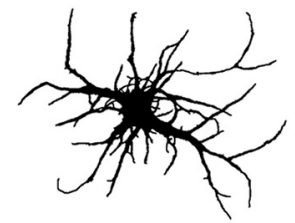

CREB S133A + BDNF

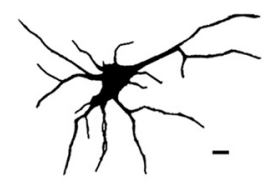

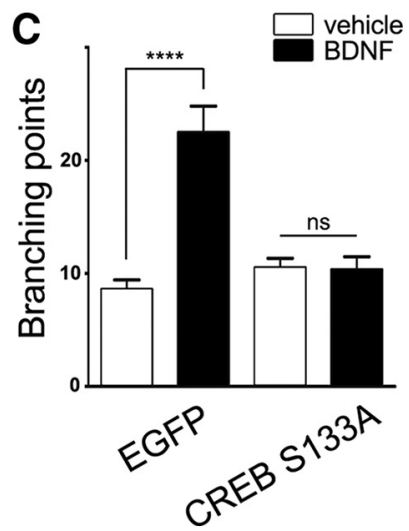

Figure 5. CREB is required for BDNF-induced dendritic branching. To confirm whether BDNF-mediated phosphorylation of CREB at S133 was critical for a cellular response, we compared BDNF-induced dendritic branching in neurons expressing either EGFP or a nonphosphorylatable mutant of CREB (S133A). Neurons were transfected after $7 \mathrm{~d}$ in vitro and treated with BDNF immediately after transfection for $48 \mathrm{~h}$. $A$, Then, immunostaining for MAP2 was used to analyze the morphology of the dendritic arbor. $\boldsymbol{B}, \boldsymbol{C}$, Sholl analysis $(\boldsymbol{B})$ and direct counting of the number of branching points $(C)$ from 18 neurons/condition in three independent experiments showed that the phosphorylation of CREB is required for BDNF-induced dendritic branching. Sholl curves were compared using two-way ANOVA and Bonferroni's multiple-comparison post-test. Branching points data were analyzed using two-way ANOVA. Significance levels for the different statistical tests are labeled ${ }^{*} p<0.05$; ${ }^{* *} p<0.01$; ${ }^{* * *} p<0.0001$; ns, nonsignificant. Scale bars, $10 \mu \mathrm{m}$. A summary of the statistics for this figure, including tests, degrees of freedom, and exact $p$ values, has been included in Extended Data Figure 5-1 supporting Figure 5.

(Egr1, Egr2, Atf3, Fos, Crem, and Junb). All of these transcription factors or transcriptional regulators are early-response genes regulated by neuronal activity, which is consistent with the short time of BDNF exposure (Flavell and Greenberg, 2008; Yap and Greenberg, 2018). Then, we analyzed whether the expression of the Rab11DN mutant alters BDNF-induced changes in gene expression. We observed a general downregulation of BDNF-responsive genes, since only Egr1 and Egr2 were still significantly upregulated by BDNF in cells expressing Rab11DN (Fig. 6B). These results were consistent with the fact that Egr1 and Egr2 were the genes most strongly upregulated by BDNF in neurons expressing EGFP (Fig. $6 A$ ). When comparing the levels of expression of BDNF-responding genes in cells expressing EGFP and Rab11DN, the expression of Egr1 was significantly lower in Rab11DN-expressing neurons (Fig. 6C). Additionally, there were three genes (S100a6, Stat3, and Nf1) that were slightly downregulated (S100a6) or upregulated (Stat3 and Nf1) by BDNF in EGFP-expressing neurons, without reaching statistical significance. However, these proteins were significantly upregulated (S100a6) or downregulated (Stat3 and Nf1) in neurons expressing Rab11DN and treated with BDNF, compared with neurons expressing EGFP and treated with BDNF (Extended Data
Fig. 6-3). This suggests that BDNF differentially regulates gene expression in Rab11DN-expressing neurons compared with control neurons (EGFPexpressing neurons).

Another gene that is strongly upregulated by BDNF is Arc, which is an early-response gene and a well known transcriptional target of BDNF that possesses CRE response elements (Kawashima et al., 2009). We used Arc upregulation induced by BDNF as a positive control of $\mathrm{BDNF} / \mathrm{TrkB}$ activation in the experiments presented in Figure 6A-C. The upregulation of $A r c$ in EGFP-expressing neurons was 5.4 -fold (Fig. $6 D$ ), which is in the same range of fold increases observed for Egr1 and Egr2 (6-fold and 5.7-fold, respectively) in neurons treated with BDNF (Fig. $6 A)$. Consistently, Rab11DN-expressing neurons displayed an attenuated response (3.3-fold; Fig. $6 D$ ), suggesting that Rab11 has a relevant role in mediating the transcriptional response to BDNF signaling.

Our data indicate that trafficking through the recycling endosomal pathway regulated by Rab11 is crucial for BDNF signaling to define a specific profile of gene expression in hippocampal neurons. Since many of the genes transcriptionally regulated by BDNF signaling and Rab11 activity are related to growth and neuronal plasticity, this finding provides a mechanism explaining how trafficking, signaling, and gene expression patterns are integrated toward all-embracing neuronal responses such as BDNF-induced remodeling of the dendritic tree.

\section{Discussion}

Neurotrophins have multiple roles in the CNS, including shaping the morphology of neurons. By using these molecules, neurons integrate local events, such as local remodeling of the cytoskeleton, with long-distance cell responses, including changes in gene expression (Matusica and Coulson, 2014). Whether the endocytic pathway is required for BDNF-mediated nuclear signaling to induce dendritic branching is unclear. We have previously shown that Rab5-Rab11 endosomes are required for BDNF-mediated dendritic branching (Lazo et al., 2013; Moya-Alvarado et al., 2018). The aim of our study was to determine whether both Rab5 and Rab11 were required for BDNF-induced CREB phosphorylation and transcriptional regulation. We found that both GTPases were required for Erk1/2-dependent CREB nuclear activation and, consistent with the role of the early and recycling pathways in regulating CREB-dependent transcription, Rab11 was also required for BDNF-induced expression changes in genes containing CRE binding sites. Altogether, our results suggest that endocytic pathways regulate the propagation of BDNF signaling to the nucleus in addition to local events reported previously (Huang et al., 2013; Lazo et al., 2013; Moya-Alvarado et al., 2018).

Signaling endosomes containing activated receptors and signaling molecules help to integrate local and distal processes (Zhou and Snider, 2006). These signaling carriers can be diverse in nature, encompassing specialized Rab5-positive early 

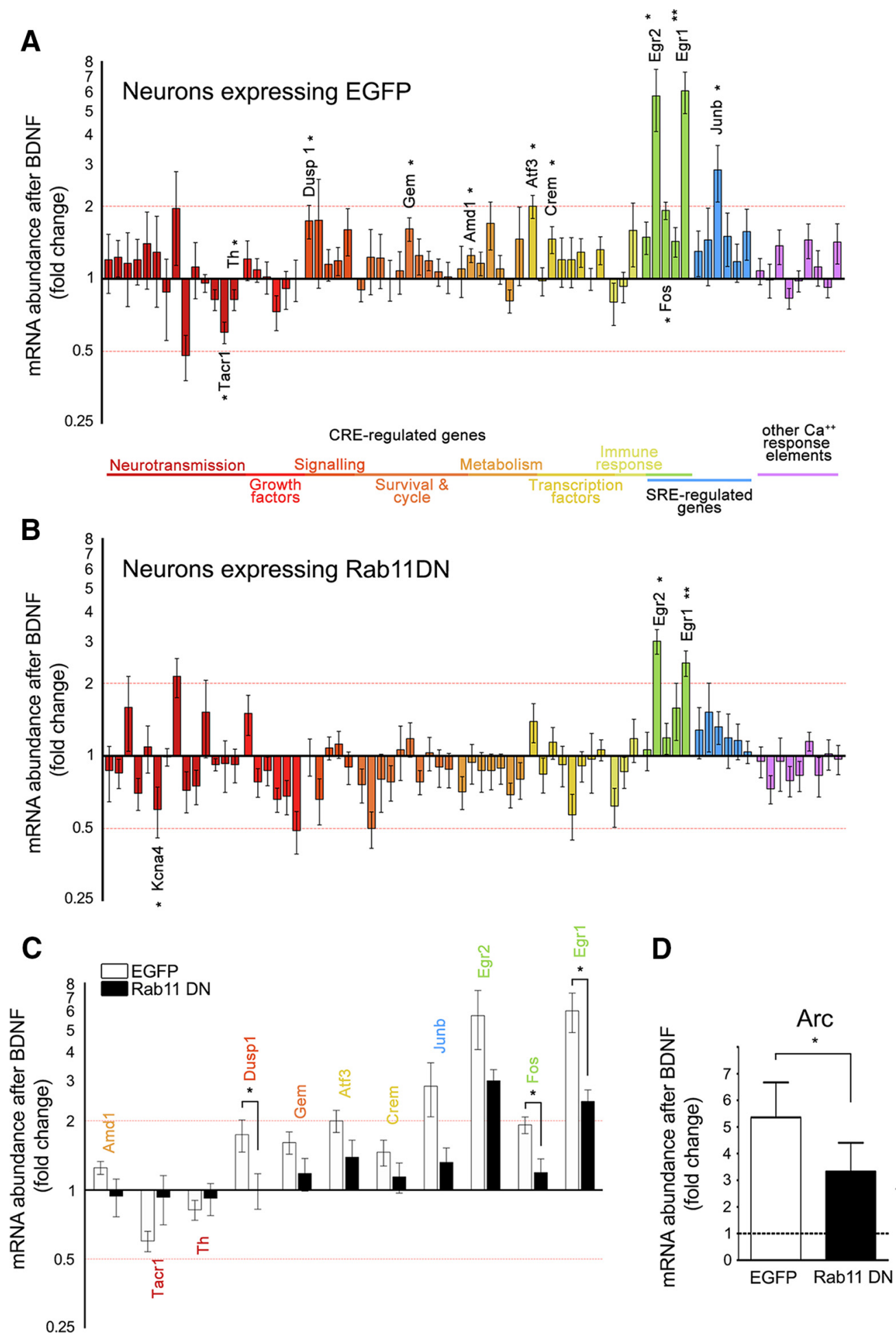

Figure 6. Rab11 activity is required for a distinctive CREB-mediated gene expression pattern in response to BDNF. A, To explore BDNF-induced changes in the expression of multiple CREBregulated genes, we used a PCR array including 84 genes with cAMP, calcium, and serum response elements. Reliable data obtained from 76 of the genes in six independent experiments were categorized by their annotated functions and plotted as a fold change in BDNF-stimulated neurons compared with nonstimulated neurons in both groups expressing EGFP, resulting in significant differences in 11 of these genes. $\boldsymbol{B}$, To study the effect of BDNF on neurons expressing Rab11DN, we studied the fold change in BDNF-stimulated to nonstimulated neurons, with both groups expressing Rab11 DN. In this case, only two genes (Egr1 and Egr2) were upregulated by BDNF, and overall, the expression of Rab11DN downregulated the BDNF response. C, To corroborate that Rab11DN expression downregulates the BDNF response, neurons expressing EGFP (control) or Rab11DN and treated with BDNF were compared. The analysis indicated an overall attenuation of the BDNF effect: 3 of the 11 genes upregulated by BDNF in $\boldsymbol{A}$ were downregulated in a statistically significant manner. $\boldsymbol{D}$, Changes in the expression of the early-response gene Arc following $4 \mathrm{~h}$ of BDNF stimulation were studied in neurons expressing control EGFP or DN mutant Rab11. Arc is not included in the commercial PCR array and was used as a positive control of BDNF response. Statistical significance of gene expression data was analyzed by using a paired Student's $t$ test. The level of significance for the different tests is indicated as follows: ${ }^{*} p<0.05 ;{ }^{* *} p<0.01$. A summary of the statistics for this figure, including tests, degrees of freedom, and exact $p$ values, has been included in Extended Data Figure 6-1 supporting Figure 6 . See Extended Data Figure 6-2 for a complete list of reliable genes. Extended Data Figure 6-3 includes the raw data and analysis step by step of the PCR array of the six replicas performed. 


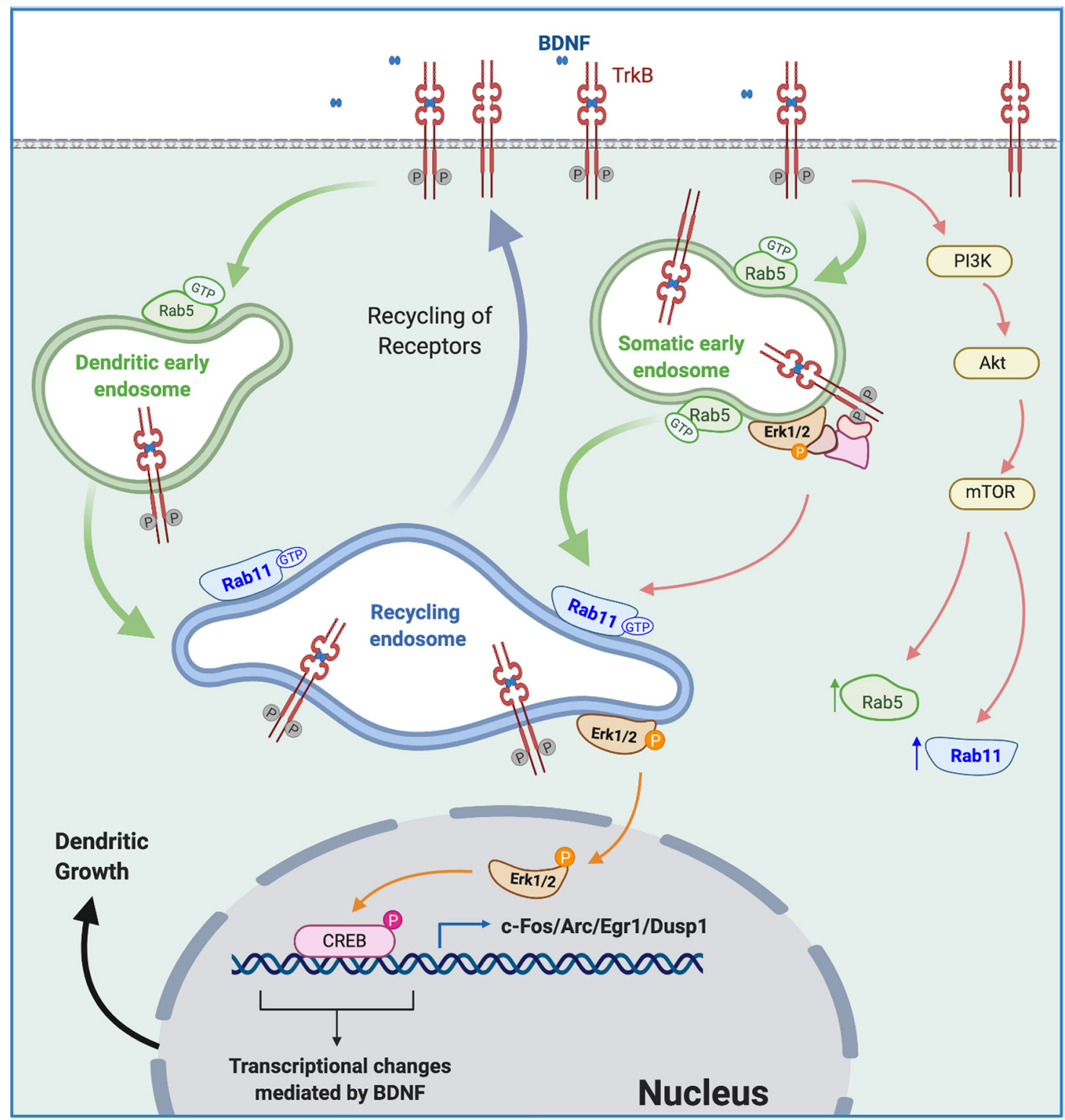

Figure 7. Model summarizing the functional relationship between Rab5-Rab11 GTPases and BDNF/TrkB-mediated CREB transcriptional regulation. In the plasma membrane, BDNF binds TrkB, promoting its activation and internalization in Rab5-positive early endosomes (in green) in dendrites (Moya-Alvarado et al., 2018) and somas of hippocampal neurons. From early endosomes, receptors are sorted to Rab11-positive recycling endosomes (in blue) or to late endosomes/lysosomes for degradation (data not shown). TrkB increases the activity of both Rab5 and Rab11 GTPases (Lazo et al., 2013; Moya-Alvarado et al., 2018), in addition to classical signaling pathways such as PI3K-Akt-mTor (in light yellow) and MAPK/Erk1/2 (in orange). The activity of Rab5 and Rab11 was required for the long-lasting activation of Akt and Erk1/2. Interestingly, reducing the activity of Rab5 diminished the levels of the endogenous Rab11 protein. Consistent with these results, the inhibition of Rab5 activity resulted in severe downregulation of Erk1/2 activation, positioning Rab5 activity upstream of Rab11 and long-lasting Erk1/2 activity. Erk1/2 was required for (REB phosphorylation in the nucleus (in pink), and reducing the activity of both Rab5 and Rab11 blocked CREB phosphorylation. Finally, blocking Rab11 activity reduced earlygene transcriptional regulation mediated by BDNF. Akt activation by BDNF may contribute to Rab5-Rab11-mediated CREB nuclear phosphorylation by increasing the protein levels of both Rab5 and Rab11 GTPases (Moya-Alvarado et al., 2018) and thus support a positive feedback loop for intracellular BDNF/TrkB signaling. Overall, our data support a role for somatic Rab11 recycling endosomes as an integrator of intracellular signaling mediated by BDNF/TrkB signaling endosomes and leading to transcriptional changes. Created with https://biorender.com.

endosomes (Wu et al., 2001a; Cui et al., 2007; Liu et al., 2007), Rab7-positive late endosomes (Deinhardt et al., 2007; Zhou et al., 2012), and Rab11-positive recycling endosomes (Ascaño et al., 2009; Barford et al., 2018), among others.

Rab11 recycling endosomes are distributed along the different domains of neurons, including the pericentriolar recycling endosomes in the soma, and tubular and vesicular endosomes in dendrites (Schmidt and Haucke, 2007) (Fig. 2C). In hippocampal neurons, BDNF stimulation results in the accumulation of Rab11 endosomes in dendrites, which explains the increase in Rab11dependent dendritic recycling of TrkB (Lazo et al., 2013) and provides a mechanism to regulate the local cytoskeleton to induce dendritic branching. On the other hand, BDNF increases the vesiculation and transport of Rab5 dendritic endosomes to the soma (Moya-Alvarado et al., 2018). It is possible that dendritic-derived Rab5 endosomes sort dendriticactivated BDNF/TrkB signaling complexes to pericentriolar Rab11 endosomes to allow integration between dendritic and nuclear signaling induced by BDNF in hippocampal neurons. Our results suggest a model in which TrkB signaling from Rab11 pericentriolar endosomes allows proper activation of an Erk1/2-CREB-mediated genetic program induced by 
BDNF; in addition, repeated rounds of TrkB recycling contribute to long-lasting BDNF signaling from Rab11 pericentriolar endosomes (Fig. 7).

Several lines of research have suggested that CREB activation requires signaling from intracellular organelles in close proximity with the nucleus. In thyroid primary cells, sustained activation of PKA from the trans-Golgi network (TGN) is required for CREB activation induced by internalized TSH receptors (Godbole et al., 2017), and the sorting of internalized receptors to the TGN requires Rab11 (Nakai et al., 2013; Welz et al., 2014). Additionally, we have previously shown that a constitutively active mutant of Rab11 allows sensitization to endogenous BDNF, increasing the phosphorylation of CREB (Lazo et al., 2013). This last observation is consistent with BDNF increasing the activity of Rab11 in dendrites as well as cell bodies of hippocampal neurons (Lazo et al., 2013) and with the requirement of Rab11 activity to promote CREB-dependent transcription (Fig. 6). Additionally, the signaling endosome effector coronin-1 was shown to be required for sympathetic neuronal survival and CREB activation in sympathetic neurons. Coronin-1 allows sorting from axonal signaling endosomes to Rab11 recycling endosomes in neuronal cell bodies, precluding lysosomal sorting and degradation of activated TrkA receptors (Suo et al., 2014). Thus, Rab11 recycling endosomes constitute a platform for the stabilization and integration of signaling receptors, avoiding degradation and allowing intracellular signaling and neuronal function.

Wu et al. (2001b) showed that sustained Erk1/2 activity was critical to induce CREB-dependent gene expression, which is in line with BDNF-induced CREB phosphorylation in the nucleus mediated by Erk1/2 in our study. Reduction of nuclear pErk1/2 by Rab5 and Rab11 DN mutants is, therefore, consistent with reduced CREB phosphorylation. Downregulation of CREB nuclear activation did not occur because of a reduction in the basal levels of the receptor or signaling molecules since $48 \mathrm{~h}$ of expression of Rab5DN and Rab11DN mutants did not preclude the activation of the receptor or the engagement of PI3K-Akt and Erk1/2 signaling pathways after $5 \mathrm{~min}$ of BDNF stimulation. However, two different techniques revealed that sustained activation of Erk1/2 (1-2 h of BDNF stimulation) was compromised in neurons expressing the mutants. The precise mechanism by which signaling endosomes facilitate the translocation of $\mathrm{pErk} 1 / 2$ to the nucleus needs further investigation; however, based on other cell models (Mebratu and Tesfaigzi, 2009), phosphorylation of Erk1/2 from intracellular organelles may promote its association with a subset of interactors that facilitate its nuclear translocation or prevent Erk1/2 from interacting with proteins that retain it in the cytoplasm. Such a mechanism has been studied for the nuclear targeting of an endosomal E3 ubiquitin ligase in response to increased PKC signaling, in which reducing the activity of Rab11 abolishes nuclear targeting of this endosomal protein (Bocock et al., 2010).

In our study, the gene expression pattern triggered by BDNF signaling was significantly attenuated when Rab11 function was abrogated. The subset of BDNF-regulated genes that depends on endocytic trafficking through the recycling pathway encompasses diverse functional groups involved in metabolism, neurotransmission, and regulation of transcription, among others. These genes include early-response genes, such as Egr1, Egr2, Fos, and $A r c$, which are important for brain plasticity and memory formation (Minatohara et al., 2015; Heroux et al., 2018). Of note, of the 64 genes analyzed containing CRE binding sites, Egr1 and Egr2 were the most highly upregulated by BDNF and downregulated by reduced Rab11 activity (Fig. $6 B, C$ ). It has been found that the increase in the transcriptional activity of Egr1 correlates with the activation of different neuronal ensembles involved in learning and memory, anxiety, drug addiction, and neuropsychiatric disorders by regulating a variety of genes involved in vesicular release and endocytosis, neurotransmitter metabolism, and actin cytoskeleton regulators, among others (Duclot and Kabbaj, 2017). Another TF and immediate-early gene regulated by CREB activation in our study was c-fos. Fos forms heterodimers with Jun to assemble the activating protein 1 complex (AP1), which regulates activity-dependent transcription in a neuronal subtypespecific manner. Indeed, it has been proposed that AP1, in addition to binding promoters, also binds enhancers, allowing chromatin remodeling and the expression of genes in a neuronal population-specific manner (Yap and Greenberg, 2018).

One interesting aspect to explore in future studies is the involvement of the BDNF-Rab5/Rab11-Erk1/2-CREB-c-fosEgr1 pathway in transcription-dependent long-term memory and dendritic morphology maintenance. In particular, the contribution of BDNF- and Rab11-mediated CREB activation to a positive feedback loop that regulates activity-dependent transcription.

While the role of Egr1-mediated transcription is well documented, the role of Egr2 has been less investigated and appears to have opposed functions compared with Egr1. Indeed, the behavior analysis of forebrain-specific Cre-mediated Egr2 deletion mice showed improved learning and memory in specific learning and memory tasks (Poirier et al., 2007). Similarly, we also found that BDNF increased the transcription of Dusp1, a phosphatase that downregulates the activation of nuclear Erk1/2 (Lawan et al., 2013; Scheffzek and Shivalingaiah, 2019). On the other hand, one of the roles associated with Arc in neurons is the endocytosis of the AMPA receptor process that is required for synaptic depression (Barylko et al., 2018). Thus, our results suggest that the BDNF-Rab5/Rab11-Erk1/2-CREB pathway also triggers genes that downregulate neurotrophic responses, providing a mechanism for homeostatic regulation. The involvement of BDNF-Rab5/Rab11-Erk1/2-CREB-regulated genes in learning and memory may explain how endosomal system abnormalities and neurotrophin receptor trafficking defects observed in neurodegenerative diseases such as Alzheimer's disease, Huntington's disease, amyotrophic lateral sclerosis, and Niemann-Pick type C disease are mechanistically linked to dendritic atrophy and synaptic plasticity loss (Cabeza et al., 2012; Liot et al., 2013; Brady and Morfini, 2017; Xu et al., 2018; Burk and Pasterkamp, 2019).

In summary, our results suggest that Erk1/2-dependent nuclear CREB phosphorylation depends on early and recycling endosomal platforms to trigger the genetic program required for dendritic arbor remodeling, among other functions. This work provides new evidence of a mechanistic link between vesicular trafficking and gene expression, reinforcing the possibility of further exploiting signaling endosomes as a tool to manipulate neuronal physiology.

\section{References}

Ascaño M, Richmond A, Borden P, Kuruvilla R (2009) Axonal targeting of Trk receptors via transcytosis regulates sensitivity to neurotrophin responses. J Neurosci 29:11674-11685.

Barford K, Deppmann C, Winckler B (2017) The neurotrophin receptor signaling endosome: where trafficking meets signaling. Dev Neurobiol 77:405-418.

Barford K, Keeler A, McMahon L, McDaniel K, Yap CC, Deppmann CD, Winckler B (2018) Transcytosis of TrkA leads to diversification of dendritic signaling endosomes. Sci Rep 8:4715.

Barylko B, Wilkerson JR, Cavalier SH, Binns DD, James NG, Jameson DM, Huber KM, Albanesi JP (2018) Palmitoylation and membrane binding of 
Arc/Arg3.1: a potential role in synaptic depression. Biochemistry 57:520524.

Bocock JP, Carmicle S, Madamba E, Erickson AH (2010) Nuclear targeting of an endosomal E3 ubiquitin ligase. Traffic 11:756-766.

Brady ST, Morfini GA (2017) Regulation of motor proteins, axonal transport deficits and adult-onset neurodegenerative diseases. Neurobiol Dis 105:273-282.

Bronfman FC, Lazo OM, Flores C, Escudero CA (2014) Spatiotemporal intracellular dynamics of neurotrophin and its receptors. Implications for neurotrophin signaling and neuronal function. Handb Exp Pharmacol 220:33-65.

Burk K, Pasterkamp RJ (2019) Disrupted neuronal trafficking in amyotrophic lateral sclerosis. Acta Neuropathol 137:859-877.

Cabeza C, Figueroa A, Lazo OM, Galleguillos C, Pissani C, Klein A, Gonzalez-Billault C, Inestrosa NC, Alvarez AR, Zanlungo S, Bronfman FC (2012) Cholinergic abnormalities, endosomal alterations and up-regulation of nerve growth factor signaling in Niemann-Pick type C disease. Mol Neurodegener 7:11.

Cha-Molstad H, Keller DM, Yochum GS, Impey S, Goodman RH (2004) Cell-type-specific binding of the transcription factor CREB to the cAMPresponse element. Proc Natl Acad Sci U S A 101:13572-13577.

Cohen MS, Bas Orth C, Kim HJ, Jeon NL, Jaffrey SR (2011) Neurotrophinmediated dendrite-to-nucleus signaling revealed by microfluidic compartmentalization of dendrites. Proc Natl Acad Sci U S A 108:1124611251

Cui B, Wu C, Chen L, Ramirez A, Bearer EL, Li WP, Mobley WC, Chu S (2007) One at a time, live tracking of NGF axonal transport using quantum dots. Proc Natl Acad Sci U S A 104:13666-13671.

Deinhardt K, Reversi A, Berninghausen O, Hopkins CR, Schiavo G (2007) Neurotrophins Redirect p75NTR from a clathrin-independent to a clathrin-dependent endocytic pathway coupled to axonal transport. Traffic 8:1736-1749.

Du K, Montminy M (1998) CREB is a regulatory target for the protein kinase Akt/PKB. J Biol Chem 273:32377-32379.

Duclot F, Kabbaj M (2017) The role of early growth response 1 (EGR1) in brain plasticity and neuropsychiatric disorders. Front Behav Neurosci 11:35.

Escudero CA, Cabeza C, Moya-Alvarado G, Maloney MT, Flores CM, Wu C, Court FA, Mobley WC, Bronfman FC (2019) c-Jun N-terminal kinase (JNK)-dependent internalization and Rab5-dependent endocytic sorting mediate long-distance retrograde neuronal death induced by axonal BDNF-p75 signaling. Sci Rep 9:6070.

Finkbeiner S, Tavazoie SF, Maloratsky A, Jacobs KM, Harris KM, Greenberg ME (1997) CREB: a major mediator of neuronal neurotrophin responses. Neuron 19:1031-1047.

Finsterwald C, Fiumelli H, Cardinaux JR, Martin JL (2010) Regulation of dendritic development by BDNF requires activation of CRTC1 by glutamate. J Biol Chem 285:28587-28595.

Flavell SW, Greenberg ME (2008) Signaling mechanisms linking neuronal activity to gene expression and plasticity of the nervous system. Annu Rev Neurosci 31:563-590.

Fu X, Yang Y, Xu C, Niu Y, Chen T, Zhou Q, Liu JJ (2011) Retrolinkin cooperates with endophilin A1 to mediate BDNF-TrkB early endocytic trafficking and signaling from early endosomes. Mol Biol Cell 22:3684-3698.

Godbole A, Lyga S, Lohse MJ, Calebiro D (2017) Internalized TSH receptors en route to the TGN induce local Gs-protein signaling and gene transcription. Nat Commun 8:443.

Goldenring JR (2015) Recycling endosomes. Curr Opin Cell Biol 35:117-122.

Gonzalez A, Moya-Alvarado G, Gonzalez-Billaut C, Bronfman FC (2016) Cellular and molecular mechanisms regulating neuronal growth by brain-derived neurotrophic factor. Cytoskeleton (Hoboken) 73:612-628.

Heroux NA, Osborne BF, Miller LA, Kawan M, Buban KN, Rosen JB, Stanton ME (2018) Differential expression of the immediate early genes c-Fos, Arc, Egr-1, and Npas4 during long-term memory formation in the context preexposure facilitation effect (CPFE). Neurobiol Learn Mem 147:128-138.

Horch HW (2004) Local effects of BDNF on dendritic growth. Rev Neurosci 15:117-129.

Horch HW, Katz LC (2002) BDNF release from single cells elicits local dendritic growth in nearby neurons. Nat Neurosci 5:1177-1184.

Huang EJ, Reichardt LF (2003) Trk receptors: roles in neuronal signal transduction. Annu Rev Biochem 72:609-642.
Huang SH, Wang J, Sui WH, Chen B, Zhang XY, Yan J, Geng Z, Chen ZY (2013) BDNF-dependent recycling facilitates TrkB translocation to postsynaptic density during LTP via a Rab11-dependent pathway. J Neurosci 33:9214-9230.

Jovic M, Sharma M, Rahajeng J, Caplan S (2010) The early endosome: a busy sorting station for proteins at the crossroads. Histol Histopathol 25:99112.

Kawashima T, Okuno H, Nonaka M, Adachi-Morishima A, Kyo N, Okamura M, Takemoto-Kimura S, Worley PF, Bito H (2009) Synaptic activity-responsive element in the Arc/Arg3.1 promoter essential for synapse-to-nucleus signaling in activated neurons. Proc Natl Acad Sci U S A 106:316-321.

Kowiański P, Lietzau G, Czuba E, Waśkow M, Steliga A, Moryś J (2018) BDNF: a key factor with multipotent impact on brain signaling and synaptic plasticity. Cell Mol Neurobiol 38:579-593.

Kwon M, Fernández JR, Zegarek GF, Lo SB, Firestein BL (2011) BDNF-promoted increases in proximal dendrites occur via CREB-dependent transcriptional regulation of cypin. J Neurosci 31:9735-9745.

Langemeyer L, Fröhlich F, Ungermann C (2018) Rab GTPase function in endosome and lysosome biogenesis. Trends Cell Biol 28:957-970.

Lawan A, Shi H, Gatzke F, Bennett AM (2013) Diversity and specificity of the mitogen-activated protein kinase phosphatase-1 functions. Cell Mol Life Sci 70:223-237.

Lazo OM, Gonzalez A, Ascaño M, Kuruvilla R, Couve A, Bronfman FC (2013) BDNF regulates Rab11-mediated recycling endosome dynamics to induce dendritic branching. J Neurosci 33:6112-6122.

Leemhuis J, Boutillier S, Barth H, Feuerstein TJ, Brock C, Nürnberg B, Aktories K, Meyer DK (2004) Rho GTPases and phosphoinositide 3-kinase organize formation of branched dendrites. J Biol Chem 279:585596.

Liot G, Zala D, Pla P, Mottet G, Piel M, Saudou F (2013) Mutant Huntingtin alters retrograde transport of TrkB receptors in striatal dendrites. J Neurosci 33:6298-6309.

Liu J, Lamb D, Chou MM, Liu YJ, Li G (2007) Nerve growth factor-mediated neurite outgrowth via regulation of Rab5. Mol Biol Cell 18:1375-1384.

Marshall CJ (1995) Specificity of receptor tyrosine kinase signaling: transient versus sustained extracellular signal-regulated kinase activation. Cell 80:179-185.

Matusica D, Coulson EJ (2014) Local versus long-range neurotrophin receptor signalling: endosomes are not just carriers for axonal transport. Semin Cell Dev Biol 31:57-63.

Mebratu Y, Tesfaigzi Y (2009) How ERK1/2 activation controls cell proliferation and cell death: is subcellular localization the answer? Cell Cycle 8:1168-1175.

Minatohara K, Akiyoshi M, Okuno H (2015) Role of immediate-early genes in synaptic plasticity and neuronal ensembles underlying the memory trace. Front Mol Neurosci 8:78.

Minichiello L (2009) TrkB signalling pathways in LTP and learning. Nat Rev Neurosci 10:850-860.

Moya-Alvarado G, Gonzalez A, Stuardo N, Bronfman FC (2018) Brainderived neurotrophic factor (BDNF) regulates Rab5-positive early endosomes in hippocampal neurons to induce dendritic branching. Front Cell Neurosci 12:493.

Murray B, Alessandrini A, Cole AJ, Yee AG, Furshpan EJ (1998) Inhibition of the p44/42 MAP kinase pathway protects hippocampal neurons in a cell-culture model of seizure activity. Proc Natl Acad Sci U S A 95:1197511980.

Nakai W, Kondo Y, Saitoh A, Naito T, Nakayama K, Shin HW (2013) ARF1 and ARF4 regulate recycling endosomal morphology and retrograde transport from endosomes to the Golgi apparatus. Mol Biol Cell 24:2570-2581.

Poirier R, Cheval H, Mailhes C, Charnay P, Davis S, Laroche S (2007) Paradoxical role of an Egr transcription factor family member, Egr2/ Krox20, in learning and memory. Front Behav Neurosci 1:6.

Riccio A, Ahn S, Davenport CM, Blendy JA, Ginty DD (1999) Mediation by a CREB family transcription factor of NGF-dependent survival of sympathetic neurons. Science 286:2358-2361.

Scheffzek K, Shivalingaiah G (2019) Ras-specific GTPase-activating proteinsstructures, mechanisms, and interactions. Cold Spring Harb Perspect Med 9:a031500.

Schmidt MR, Haucke V (2007) Recycling endosomes in membrane traffic. Biol Cell 99:333-342. 
Stenmark H (2009) Rab GTPases as coordinators of vesicle traffic. Nat Rev Mol Cell Biol 10:513-525.

Suo D, Park J, Harrington AW, Zweifel LS, Mihalas S, Deppmann CD (2014) Coronin-1 is a neurotrophin endosomal effector that is required for developmental competition for survival. Nat Neurosci 17:36-45.

Tapley P, Lamballe F, Barbacid M (1992) K252a is a selective inhibitor of the tyrosine protein kinase activity of the trk family of oncogenes and neurotrophin receptors. Oncogene 7:371-381.

Villarroel-Campos D, Schiavo G, Lazo OM (2018) The many disguises of the signalling endosome. FEBS Lett 592:3615-3632.

Welz T, Wellbourne-Wood J, Kerkhoff E (2014) Orchestration of cell surface proteins by Rab11. Trends Cell Biol 24:407-415.

Wu C, Lai CF, Mobley WC (2001a) Nerve growth factor activates persistent Rap1 signaling in endosomes. J Neurosci 21:5406-5416.

Wu GY, Deisseroth K, Tsien RW (2001b) Spaced stimuli stabilize MAPK pathway activation and its effects on dendritic morphology. Nat Neurosci 4:151-158.

Xu C, Fu X, Zhu S, Liu JJ (2016) Retrolinkin recruits the WAVE1 protein complex to facilitate BDNF-induced TrkB endocytosis and dendrite outgrowth. Mol Biol Cell 27:3342-3356.
Xu W, Fang F, Ding J, Wu C (2018) Dysregulation of Rab5-mediated endocytic pathways in Alzheimer's disease. Traffic 19:253-262.

Yamashita N, Kuruvilla R (2016) Neurotrophin signaling endosomes: biogenesis, regulation, and functions. Curr Opin Neurobiol 39:139-145.

Yap EL, Greenberg ME (2018) Activity-regulated transcription: bridging the gap between neural activity and behavior. Neuron 100:330-348.

Zhang X, Odom DT, Koo S-H, Conkright MD, Canettieri G, Best J, Chen H, Jenner R, Herbolsheimer E, Jacobsen E, Kadam S, Ecker JR, Emerson B, Hogenesch JB, Unterman T, Young RA, Montminy M (2005) Genomewide analysis of cAMP-response element binding protein occupancy, phosphorylation, and target gene activation in human tissues. Proc Natl Acad Sci U S A 102:4459-4464.

Zheng J, Shen W-H, Lu T-J, Zhou Y, Chen Q, Wang Z, Xiang T, Zhu Y-C, Zhang C, Duan S, Xiong Z-Q (2008) Clathrin-dependent endocytosis is required for TrkB-dependent Akt-mediated neuronal protection and dendritic growth. J Biol Chem 283:13280-13288.

Zhou B, Cai Q, Xie Y, Sheng ZH (2012) Snapin recruits dynein to BDNFTrkB signaling endosomes for retrograde axonal transport and is essential for dendrite growth of cortical neurons. Cell Rep 2:42-51.

Zhou FQ, Snider WD (2006) Intracellular control of developmental and regenerative axon growth. Philos Trans R Soc Lond B Biol Sci 361:1575-1592. 